\title{
Indicators Related to Theorizing Measurement (A Capacity Measurement Framework)
}

\author{
Mohammad Hassanzadeh ${ }^{1}$, Hamid Reza Mahmoodi \\ ${ }^{1}$ Department of Knowledge and Information Science \\ Tarbiat Modares University \\ Tehran, Iran \\ ${ }^{2} \mathrm{Msc}$ in $\mathrm{KIS}$ \\ Tarbiat Modares University \\ Tehran, Iran \\ \{Hamidreza8520@gmail.com\}
}

\begin{abstract}
Purpose: Relatively much research has been done on theorizing and its importance. However, the number of studies related to the understanding of contexts is very negligible and so far no framework has been provided including indicators and evaluation methods. This research has been done with the aim of achieving the indicators related to theorizing and subsequently presenting a formula to measure the potential and theorizing capacity of scientific institutes.
\end{abstract}

Methodology: Library and field methods have been used to collect information. Data were first collected through a checklist and then through the AHP questionnaire. Questionnaire was distributed among experts and AHP method was used to analyze the data. Expert Choice software was used to analyze the data obtained from the AHP questionnaire.

Findings: The results indicate that individual index is 9 times more important than non-individual index in the theorizing process.

Conclusion: Although theorizing is done by a researcher or a group of researchers, but in the end, it is a collective matter or at least, several components are involved in its formation. Theorizing is a coherent, dynamic, purposeful and thoughtful practice whose results can lead researchers to recognize the credible generalizable relationship between causes and effects. Finally, the formula $T p=(0.9 I+0.1 N I)$ was presented, which can be used to assess the capacity of theorizing in institutes.

Keywords: Theory, Theorizing, Theorizing Capacity, Assessing Theorizing

DOI: https://doi.org/10.6025/stm/2020/2/72-76

\section{Introduction}

Many definitions of theory are available; each of the experts has defined the theory from their own point of view. "Theory is a set of propositions or theorems that provide explanation or description or understanding between different topics" (Harsij, 2011). According to Dubin, "theory is a set of causal relationships between phenomena" (Danaeifar, 2010). Some people believe that due to its systematic nature, is very effective and helps the researcher in achieving and understanding complex theorems (Van de Van, 1989; quoted in Najjari, 2011). Most researchers understand the importance of theory and theorizing; Researchers and experts emphasize its importance by mentioning the benefits of theorizing. Theory, by explaining, interpreting and predicting events, leads to the formulation of scientific laws and the presentation of a body in which phenomena make sense (Piroozbakht, 2013). It is not clear what indicators are important for burgeon of the theorizing process. Also according to the initial evidence, it can be argued that it is not clear what extent scientific institutes have the capacity and potentiality for theorizing. Identifying indicators related to theorizing can be a useful and efficient tool for universities and scientific institutes. Universities and scientific centers can identify their strengths and weaknesses by matching their status and facilities with theorizing indicators and also by computing the capacity of theorizing and try to strengthen the strengths and eliminate the weaknesses. The main problem of this research is what are the indicators related to theorizing and how can the theorizing 
capacity of scientific centers be calculated? In the field of identifying indicators related to theorizing, so far no research has been done that has directly focused on this problem, but indirectly, some researches has been done that have been briefly mentioned.Previous researches have tended to examine the barriers of theorizing and research. In fact, researchers have focused less on identifying the indicators and factors influencing theorizing. But a logical point to note here is that sometimes the presence or absence or even having or not having of a trait can be effective in referring to that trait as a promoter or deterrent.Indeed, having or not having something makes us consider it a promoter (effective factor) or a deterrent (obstacle). For example, in the field of educational accomplishment, intelligence seems to be an important component. But the truth is that intelligence is not in itself one of the factors influencing educational accomplishment, but "having or not having intelligence" makes us consider it an obstacle or a factor. This is also true in the field of theorizing. For example, the individual dimension is one of the dimensions that have been identified as a barrier of theorizing. Paying attention to the internal components of the individual dimension shows that shifting the verbs of "having and not having" can change the role of a component from an obstacle (deterrent) to a positive factor (promoter). For example, lack of or weakness in critical thinking is one of the individual barriers. Naturally, having critical thinking can be considered a positive individual factor. Mahmoodi, Hassanzadeh and Zandian (2019) in a qualitative study focused on modeling the barriers of theorizing in information science in Iranian universities concluded that individual, educational, cultural, managerial, economic, social and political barriers prevent the researcher from theorizing. The model of the septet barriers of theorizing shows that in the individual dimension, components such as weakness in critical thinking, self-confidence and creativity can take the researcher away from theorizing. The results indicate that the researcher is at the center of theorizing and ignoring theorizing is more relevant to the researcher than other dimensions.Ashrafi et al. (2015) examined the individual, socio-cultural, and organizational dimensions of research barriers. Their results show that students' course density is more deterrent than other barriers. The individual dimension seems to be more influential than other dimensions. According to them, if a person is not familiar with the research method, he will not be very successful in research. These results can be generalized to theorizing. In fact, it seems that a person who is not familiar with research methodologies and theorizing will not succeed in this. Alexander Olasen et al. (2017) examined research barriers in the emergency medical group. The findings of this study indicate that time; skill and culture are three important factors in research. In other words, a person who does not have enough time and skills to conduct research and theorizing will be less interested in this. Also, if the culture of research and theorizing is not institutionalized in the scientific community, we cannot hope to conduct research and provide consistent theories. Aditi Hegde et al. (2017) in a study found that workload, time constraints, lack of financial resources, weakness in education and lack of instructor change the research process. When the workload of researchers as well as theorists is large, conducting research will be difficult. Most researchers believe that time constraints prevent them from conducting research.In addition, a researcher who is not financially supported and has financial concerns will not pay attention to research and theorizing.

According to previous research, it can be concluded that barriers are generally related either to the researcher or to the public and scientific society, which are considered non-individual barriers. In other words, sometimes the researcher does not have enough motivation and potency to do theorizing and sometimes the managerial, economic, and cultural systems governing the scientific society hinder theorizing. In this research, it has been endeavored to identify the indicators related to theorizing measurement by reviewing previous researches and documents with the aim of presenting a formula for measuring theorizing capacity.

\section{Conceptual Framework}

In this research, the model of septet barriers of theorizing of Mahmoodi, Hassanzadeh and Zandian (2020) is considered as the initial conceptual framework. The septet dimensions of this model, including individual, educational, cultural, managerial, economic, social and political dimensions, were used to achieve a formula. In this model, the researcher as the main entity is at the center of the theorizing process and the relationships of other dimensions around the individual dimension are explained. The main idea of the concepts of this research is also based on the model of septet barriers. In fact, in the process of theorizing, on the one hand, the researcher and on the other hand, the (non-individual) society plays a role.

This is an applied research and library and field methods were used to collect information. In order to collect information, checklist tools and a questionnaire designed by AHP method were used. The AHP questionnaire consists of 31 questions. In order to answer the questions, the experts were asked to give score the four elements $A, B, C$ and $D$ from 1 to 9 . The elements were placed opposite each other in pairs (Table 1).

The questionnaire was distributed between the experts. In order to measure the validity and reliability of the questionnaire, the inconsistency rate was used. The inconsistency rate is an indicator whose value indicates possible inconsistencies in the pairwise comparison matrix. If inconsistency rate would be less than 0.1 , the compatibility of the comparison matrix is approved and acceptable. However, if the inconsistency rate would be greater than 0.1 , it indicates inconsistency and instability in the evaluations and judgments of experts. In this study, all indicators and questions had acceptable compatibility. Expert Choice 11 software was used to analyze the data. 


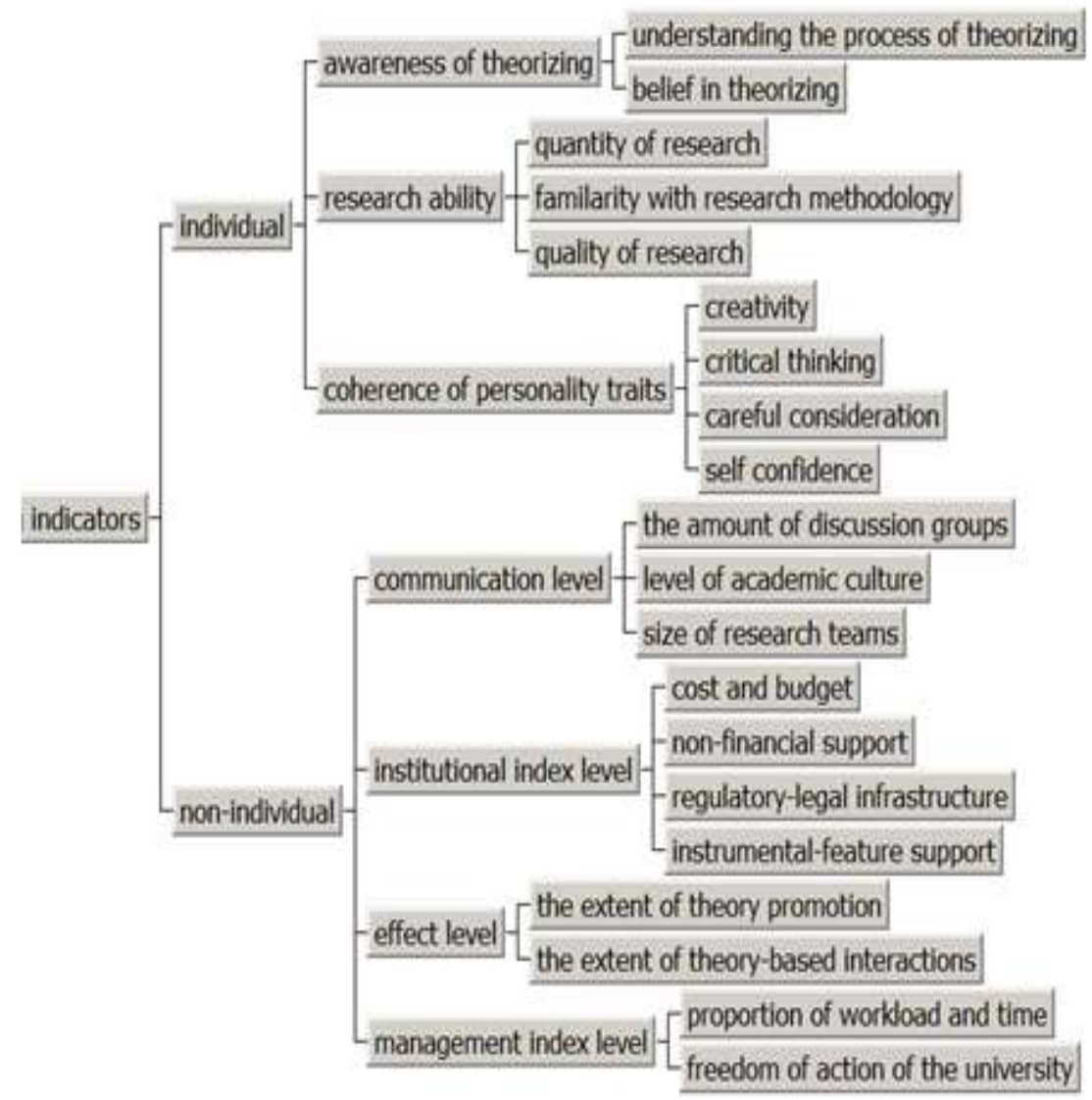

Figure 1. Conceptual model

Table 1. Guide to scoring questions (scores: identical 1 , slightly more important 3 , more important 5 , relatively important 7 , extremely important 9 , numbers $2,4,6,8$ intermediate)

\begin{tabular}{|l|l|lllllllllllllllll|l|}
\hline $\mathrm{B}$ & 9 & 8 & 7 & 6 & 5 & 4 & 3 & 2 & 1 & 2 & 3 & 4 & 5 & 6 & 7 & 8 & 9 & $\mathrm{~A}$ \\
\hline $\mathrm{C}$ & 9 & 8 & 7 & 6 & 5 & 4 & 3 & 2 & 1 & 2 & 3 & 4 & 5 & 6 & 7 & 8 & 9 & $\mathrm{~A}$ \\
\hline $\mathrm{D}$ & 9 & 8 & 7 & 6 & 5 & 4 & 3 & 2 & 1 & 2 & 3 & 4 & 5 & 6 & 7 & 8 & 9 & $\mathrm{~A}$ \\
\hline $\mathrm{C}$ & 9 & 8 & 7 & 6 & 5 & 4 & 3 & 2 & 1 & 2 & 3 & 4 & 5 & 6 & 7 & 8 & 9 & $\mathrm{~B}$ \\
\hline $\mathrm{D}$ & 9 & 8 & 7 & 6 & 5 & 4 & 3 & 2 & 1 & 2 & 3 & 4 & 5 & 6 & 7 & 8 & 9 & $\mathrm{~B}$ \\
\hline $\mathrm{D}$ & 9 & 8 & 7 & 6 & 5 & 4 & 3 & 2 & 1 & 2 & 3 & 4 & 5 & 6 & 7 & 8 & 9 & $\mathrm{C}$ \\
\hline
\end{tabular}

\section{Findings}

The obtained coefficients for each of the studied indicators and sub-indices have provided a suitable framework for calculating and explaining the "theorizing capacity" of scientific and academic institutions. Based on the performed formulation, the formula TP $=0.9 I+0.1 N I$ is presented. (The full name of each symbol is given in Table 12):

$I=(0.125 \mathrm{At}+0.125 \mathrm{Ra}+0.75 \mathrm{Cpt})$ 


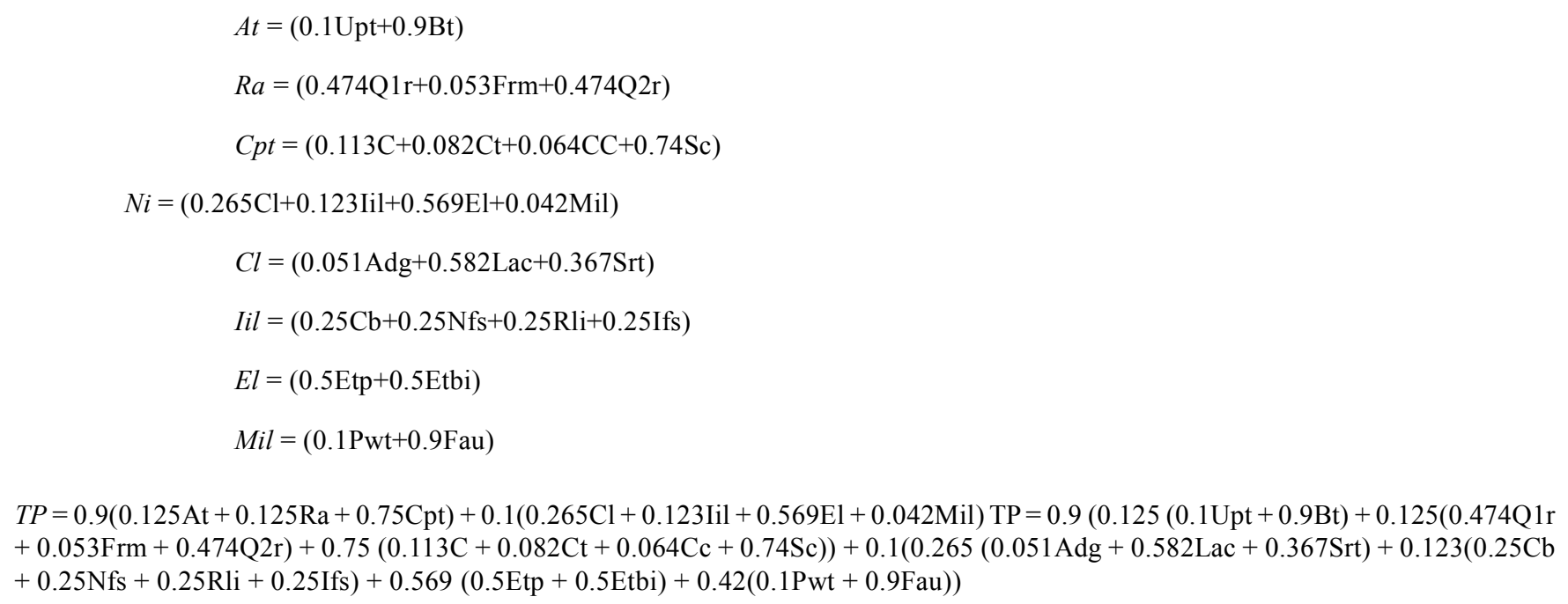

Table 2. Indicators and symbols (abbreviations)

\begin{tabular}{|l|l|}
\hline Indicators sub indicators & Symbol \\
\hline Theorizing potential & $\mathrm{T}-\mathrm{p}$ \\
Individual & $\mathrm{IS}$ \\
Non-Individual & $\mathrm{NI}$ \\
Awareness of theorizing & $\mathrm{At}$ \\
Research ability & $\mathrm{Ra}$ \\
Coherence of personality traits & $\mathrm{Cpt}$ \\
Communication level & $\mathrm{Cl}$ \\
Institutional index level & $\mathrm{Iil}$ \\
Effect level & $\mathrm{El}$ \\
Management index level & $\mathrm{Mil}$ \\
Understanding the process of theorizing & $\mathrm{Upt}$ \\
Belief in theorizing & $\mathrm{Bt}$ \\
Quantity of research & $\mathrm{Q} 1 \mathrm{r}$ \\
Familiarity with research methodology & $\mathrm{Frm}$ \\
Quality of research & $\mathrm{Q} 2 \mathrm{r}$ \\
Creativity & $\mathrm{C}$ \\
Critical thinking & $\mathrm{Ct}$ \\
Careful consideration & $\mathrm{Cc}$ \\
Self confidence & $\mathrm{Adg}$ \\
The amount of discussion groups & $\mathrm{Sc}$ \\
Level of academic culture & . \\
\hline
\end{tabular}


Cost and budget

Non-financial support

Regulatory-legal infrastructure

Instrumental-feature support

The extent of theory promotion

The extent of theory-based interactions

Proportion of workload and time

Freedom of action of the university
$\mathrm{Cb}$

$\mathrm{Nfs}$

Rli

Ifs

Etp

Etbi

Pwt

Fau

\section{Conclusion}

According to the findings, it proposes a new branch of Theorometrics. Due to time constraints and the need for further validation, this research will continue. According to new findings, it is possible to refine the formula. In the next steps, a benchmark should be designed for each of the introduced indicators. Therefore, although the formula itself has good computational capabilities, providing an accurate picture of a research institute or university requires repeated reviews and possible corrections to the formula. In the following, on the one hand, the indicators will be completed and on the other hand, more accurate metrics for the indicators will be introduced.It should be emphasized that the capacity for theorizing can vary in different disciplines, institutions and even in different countries. For example, indicators such as politics in different disciplines have different functions. The definition and placement of policy-related indicators, such as political intervention in science and the toxic scientific atmosphere, are less relevant in disciplines such as computer science, literature, and so on. In the social sciences, however, presenting theories that contradict national, regional, and global beliefs can be challenging. Therefore, the intensity and weakness of political indicators are not the same in different fields, and therefore efforts to classify, coefficient allocation and refine them will continue.

\section{References}

[1] Ashrafi, H., Zarmehr, F., GhazaviKhorasgani, Z., Kazempour, Z., Taebi Imani, S.(2015).Barriers to Research Activities from the Perspective of the Student of Isfahan University of Medical Sciences. Acta Inform Med, 23(3), $155-159$.

[2] Dubin, R. (In press), Theorizing, translated by Hassan Danaeifar.

[3] Harsij, H. (2011). Barriers to Theorizing, Methodology, Science Production; Obstacles and solutions [interview] (October 2011).

[4] Hegde, A., et. Al (2017). Attitudes, experiences, and barriers to research and publishing among dental postgraduate students of Bengaluru City: A cross-sectional study. J Indian Assoc Public Health Dent [serial online] [cited 2019 Dec 10];15:15761. Available from: http://www.jiaphd.org/text.asp?2017/15/2/157/207907.

[5] Mahmoodi, H. R., Hassanzadeh, M., Zandian, F. (2019). Modeling Barriers of Knowledge and Information Science Theorizing from the Perspective of Faculty Members of Universities of Iran. Master Thesis. Knowledge and Information Science Department. Faculty of Management and Economics. Trabiat Modares University.

[6] Najari, R. (2011). The method of theorizing of Martyr MortezaMotahhari on the subject of growth. Scientific-Research of Islamic Management. 19 (2), p.89.

[7] Olaussen, A., et. al. (2017) Barriers to conducting research: A survey of trainees in emergency medicine: EMERGENCY MEDICINE RESEARCH BARRIERS. EMA 29(2).

[8] Piruzbakht, M. (2013). Academic Counseling. [Online] [Accessed on 9/5/1396] from:http://pirouzbakht.parsiblog.com/Posts/ 2/ 